\title{
Angular dependence of upper critical field in two-band Ginzburg-Landau theory
}

\author{
I.N. Askerzade ${ }^{a, b, *}$, B. Tanatar ${ }^{b}$ \\ ${ }^{a}$ Institute of Physics, Azerbaijan National Academy of Sciences, Baku Az1143, Azerbaijan, Turkey \\ ${ }^{\mathrm{b}}$ Department of Physics, Bilkent University, 06800 Bilkent, Ankara, Turkey
}

Received 29 November 2006; received in revised form 11 March 2007; accepted 17 April 2007

Available online 3 May 2007

\begin{abstract}
Generalization of two-band Ginzburg-Landau (GL) theory to the case of anisotropic mass is presented. The temperature dependence of the anisotropy parameter of upper critical field $\gamma_{\mathrm{c} 2}(T)=H_{\mathrm{c} 2}^{\|}(T) / H_{\mathrm{c} 2}^{\perp}(T)$ and angular dependence of $H_{\mathrm{c} 2}(\theta, T)$ are calculated using anisotropic mass two-band Ginzburg-Landau theory of superconductors. It is shown that, with decreasing temperature anisotropy parameter $\gamma_{\mathrm{c} 2}(T)$ is increased. Results of our calculations are in agreement with experimental data for single crystal $\mathrm{MgB}_{2}$.

(C) 2007 Published by Elsevier B.V.
\end{abstract}

PACS: 74.20.De; 74.25.Ha; 74.70.Ad; 74.20.Mn

Keywords: Two-band superconductivity; Angular dependence; Upper critical field; Mass anisotropy

\section{Introduction}

Recently discovered [1] superconducting compound $\mathrm{MgB}_{2}$ has led to a growing amount of both experimental and theoretical works due to the fact that it holds the highest superconducting transition temperature of about $T_{\mathrm{c}}=39 \mathrm{~K}$ for a binary compound of a relatively simple crystal structure. Calculations of the band structure and the phonon spectrum predict a double energy gap [2,3], a larger gap attributed to two-dimensional $p_{x-y}$ orbitals $(\sigma-$ band) and smaller gap attributed to three-dimensional $p_{z}$ bonding and anti-bonding orbitals ( $\pi$-band). As a superconductor the electron-phonon mechanism of superconductivity [4] in $\mathrm{MgB}_{2}$ involves giant anharmonicity and nonlinear electron-phonon coupling [5]. Two-band characteristic of the superconducting state in $\mathrm{MgB}_{2}$ is clearly evident in the recently performed tunneling measurements $[6,7]$ and specific heat measurement [8]. Another class of

\footnotetext{
${ }^{*}$ Corresponding author. Address: Institute of Physics, Azerbaijan National Academy of Sciences, Baku Az1143, Azerbaijan, Turkey.

E-mail address: iasker@science.ankara.edu.tr (I.N. Askerzade).
}

two-band superconductors are the nonmagnetic borocarbides [9] $\mathrm{Lu}(\mathrm{Y}) \mathrm{Ni}_{2} \mathrm{~B}_{2} \mathrm{C}$.

Magnetic phase diagram for bulk samples of $\mathrm{MgB}_{2}$ and nonmagnetic borocarbides $\mathrm{Lu}(\mathrm{Y}) \mathrm{Ni}_{2} \mathrm{~B}_{2} \mathrm{C}$ has been of interest to researchers. In contrast to common superconductors, the upper critical field for bulk samples of $\mathrm{MgB}_{2}$ and borocarbides $\mathrm{Lu}(\mathrm{Y}) \mathrm{Ni}_{2} \mathrm{~B}_{2} \mathrm{C}$ have a positive curvature near $T_{\mathrm{c}}$. To understand the nature of the unusual behavior at a microscopic level, a two-band Eliashberg model of superconductivity was first proposed by Shulga et al. [9] for $\mathrm{LuNi}_{2} \mathrm{~B}_{2} \mathrm{C}$ and $\mathrm{YNi}_{2} \mathrm{~B}_{2} \mathrm{C}$ and recently [10] for $\mathrm{MgB}_{2}$. Two-band Ginzburg-Landau (GL) model for bulk $\mathrm{MgB}_{2}$ was successfully applied to fit the experimental results of the temperature dependence of upper and lower critical fields for $\mathrm{MgB}_{2}$ and nonmagnetic borocarbides [11-13].

Systematic deviation from single-band anisotropic GL behavior was observed in recent experimental works (see below) on angular dependence of upper critical field in $\mathrm{MgB}_{2}$ single crystals. It is necessary to take into account different characteristics of anisotropy in different bands. Motivated by these experiments, in this paper we extend our previous analysis of the two-band effects [11-13] on 
angular dependence of the upper critical field $H_{\mathrm{c} 2}(\theta, T)$. We also study the temperature dependence of anisotropy parameter $\gamma_{\mathrm{c} 2}=H_{\mathrm{c} 2}^{\|} / H_{\mathrm{c} 2}^{\perp}$ of upper critical field $H_{\mathrm{c} 2}$ in single crystals of $\mathrm{MgB}_{2}$. Within the two-band GL theory our calculations yield good agreement with experiments on the angle dependence of $H_{\mathrm{c} 2}(\theta, T)$.

The rest of this paper is organized as follows. In the next section, we outline the two-band Ginzburg-Landau theory and derive the expressions for the upper critical field $H_{\mathrm{c} 2}(T)$. In Section 3, we concentrate on the angle dependence of $H_{\mathrm{c} 2}$ and obtain expressions valid in the vicinity of the critical temperature $T_{\mathrm{c}}$. Our results for $\mathrm{MgB}_{2}$ are presented in Section 4 and discussed in the light of available experimental data.

\section{Basic equations}

In the presence of two order parameters $\Psi_{1}$ and $\Psi_{2}$ in a superconductor, GL free energy functional $\mathscr{F}$ can be written as [11-13]

$\mathscr{F}\left[\Psi_{1}, \Psi_{2}\right]=\int \mathrm{d}^{3} r\left(F_{1}+F_{12}+F_{2}+H^{2} / 8 \pi\right)$,

with

$F_{i}=\frac{\hbar^{2}}{4 m_{i}}\left|\left(\nabla-\frac{2 \pi \mathrm{i} \vec{A}}{\Phi_{0}}\right) \Psi_{i}\right|^{2}+\alpha_{i}(T) \Psi_{i}^{2}+\frac{\beta_{i}}{2} \Psi_{i}^{4}$

and

$$
\begin{aligned}
F_{12}= & \varepsilon\left(\Psi_{1} \Psi_{2}^{*}+\text { c.c. }\right) \\
& +\varepsilon_{1}\left[\left(\nabla+\frac{2 \pi \mathrm{i} \vec{A}}{\Phi_{0}}\right) \Psi_{1}^{*}\left(\nabla-\frac{2 \pi \mathrm{i} \vec{A}}{\Phi_{0}}\right) \Psi_{2}+\text { c.c. }\right] .
\end{aligned}
$$

In the above equations, $m_{i}$ denotes the effective mass of the carriers belonging to band $i(i=1,2), F_{i}$ is the free energy of the $i$ th band, and $\Phi_{0}=h c / 2 e$ is the flux quantum. The coefficient $\alpha$ is given as $\alpha_{i}=\gamma_{i}\left(T-T_{\mathrm{c} i}\right)$, which depends on temperature linearly, $\gamma_{i}$ is the proportionality constant, while the coefficient $\beta$ is independent of temperature. $\vec{H}$ is the external magnetic field related to the vector potential $\vec{A}$ by $\vec{H}=\nabla \times \vec{A}$. The quantities $\varepsilon$ and $\varepsilon_{1}$ describe interband interaction of two order parameters and their gradients, respectively. Intergradient interaction term is equal to zero in the free energy employed by Zhitomirsky and Dao [14]. However, the intergradient term as introduced by Doh et al. [15] and Affleck et al. [16] seems to be crucial. As shown by Askerzade [11-13] presence of this term leads to measurable effects in the study of $H_{\mathrm{c} 1}$ and $H_{\mathrm{c} 2}$. For instance, the effect of positive curvature in $H_{\mathrm{c} 2}$ is enhanced due to the inclusion of intergradient interaction term. In a very recent work [17] it is shown that this term is also important in the case of inclusion of anisotropic order parameters.

Minimization of the free energy functional with respect to the order parameters yields GL equations for two-band superconductors with the choice $\vec{A}=(0, H x, 0)$

$$
\begin{gathered}
-\frac{\hbar^{2}}{4 m_{1}}\left(\frac{\mathrm{d}^{2}}{\mathrm{~d} x^{2}}-\frac{x^{2}}{l_{\mathrm{s}}^{4}}\right) \Psi_{1}+\alpha_{1}(T) \Psi_{1}+\varepsilon \Psi_{2} \\
+\varepsilon_{1}\left(\frac{\mathrm{d}^{2}}{\mathrm{~d} x^{2}}-\frac{x^{2}}{l_{\mathrm{s}}^{4}}\right) \Psi_{2}+\beta_{1} \Psi_{1}^{3}=0, \\
-\frac{\hbar^{2}}{4 m_{2}}\left(\frac{\mathrm{d}^{2}}{\mathrm{~d} x^{2}}-\frac{x^{2}}{l_{\mathrm{s}}^{4}}\right) \Psi_{2}+\alpha_{2}(T) \Psi_{2}+\varepsilon \Psi_{1} \\
+\varepsilon_{1}\left(\frac{\mathrm{d}^{2}}{\mathrm{~d} x^{2}}-\frac{x^{2}}{l_{\mathrm{s}}^{4}}\right) \Psi_{1}+\beta_{2} \Psi_{2}^{3}=0,
\end{gathered}
$$

where $l_{\mathrm{s}}^{2}=\hbar c / 2 e H$ is the square of the so-called magnetic length. In the derivation of the GL equations above, small spatial variation of the gap function is assumed. Thus, the higher order derivatives are not significant in the calculation of upper critical field. As shown by Zhitomirsky and Dao [14] higher order derivatives become important for the study of the orientation of the vortex lattice along $c$-axis in $\mathrm{MgB}_{2}$ crystals. In this work, sixth order gradient terms were included to the free energy functional.

For the calculation of upper critical field $H_{\mathrm{c} 2}$, the system of Eqs. (4a) and (4b) can be linearized in the vicinity of $T_{\mathrm{c}}$ and solved using the ansatz [11] $\Psi_{1,2} \propto \mathrm{e}^{-x^{2} / 2 l_{\mathrm{s}}^{2}}$. Equation that determines the upper critical field in the isotropic case has the form

$$
\left(\frac{e H \hbar}{2 m_{1} c}+\alpha_{1}(T)\right)\left(\frac{e H \hbar}{2 m_{2} c}+\alpha_{2}(T)\right)=\left(\varepsilon-\varepsilon_{1} \frac{2 e H}{\hbar c}\right)^{2}
$$

and the solution for $H_{\mathrm{c} 2}(T)$ can be written as

$$
H_{\mathrm{c} 2}(T)=\frac{\Phi_{0}}{2 \pi \xi^{2}},
$$

where the coherence length $\xi$ of two-band superconductors is given by the expression

$$
\begin{aligned}
\xi^{2}= & \frac{\hbar^{2}}{4}\left[-\left(m_{1} \alpha_{1}(T)+m_{2} \alpha_{2}(T)+\frac{8 \varepsilon \varepsilon_{1} m_{1} m_{2}}{\hbar^{2}}\right)\right. \\
& \left.+\sqrt{\left(m_{1} \alpha_{1}(T)+m_{2} \alpha_{2}(T)+\frac{8 \varepsilon \varepsilon_{1} m_{1} m_{2}}{\hbar^{2}}\right)^{2}-4 m_{1} m_{2}\left(\alpha_{1}(T) \alpha_{2}(T)-\varepsilon^{2}\right)}\right]^{-1} .
\end{aligned}
$$

In the vicinity of the critical temperature $T_{\mathrm{c}}$, we may neglect terms of order $H^{2}$ in Eq. (5) and obtain the approximate expression for the upper critical field

$H_{\mathrm{c} 2}(T) \approx \frac{2 c}{e \hbar} \frac{\left(\varepsilon^{2}-\alpha_{1}(T) \alpha_{2}(T)\right)}{\left(\frac{\alpha_{1}(T)}{m_{2}}+\frac{\alpha_{2}(T)}{m_{1}}+\frac{8 \varepsilon \varepsilon_{1}}{\hbar^{2}}\right)}$.

We note [11-13] that the critical temperature $T_{\mathrm{c}}$ of a twoband superconductor as a result of inter-band interaction is higher than $T_{\mathrm{c} 1}$ and $T_{\mathrm{c} 2}$, i.e., $\left(T_{\mathrm{c}}-T_{\mathrm{c} 1}\right)\left(T_{\mathrm{c}}-T_{\mathrm{c} 2}\right)=$ $\varepsilon^{2} / \gamma_{1} \gamma_{2}$. 


\section{Angular effects}

Let us now consider the out-of-plane behavior of the upper critical field. In the single-band GL theory [18], when the magnetic field $H$ is tilted from the $c$-axis by an angle $\theta$, the upper critical field has an elliptic angular dependence

$H_{\mathrm{sbGL}}(\theta, T)=\frac{H_{\mathrm{c} 2}^{\perp}}{\sqrt{\cos ^{2} \theta+\gamma_{\mathrm{c} 2}^{-2} \sin ^{2} \theta}}$,

where $H_{\mathrm{c} 2}^{\|}$and $H_{\mathrm{c} 2}^{\perp}$ are the parallel and perpendicular components of $\vec{H}_{\mathrm{c} 2}$, respectively, and their ratio $\gamma_{\mathrm{c} 2}=H_{\mathrm{c} 2}^{\|} / H_{\mathrm{c} 2}^{\perp}$ is a constant independent of temperature. It is determined by the anisotropy of the effective mass in single-band superconductors

$\gamma_{\mathrm{c} 2}=\sqrt{\frac{m^{c}}{m}}$,

where $m^{c}$ is the effective mass in $c$-direction, and $m$ is the effective mass in $a b$-plane. Experimental works on upper critical field in $\mathrm{MgB}_{2}$ have shown that not only $\gamma_{\mathrm{c} 2}$ changes with temperature [19-23], but deviation from the elliptic angular dependence given in Eq. (7) grows with increasing temperature. Theoretical calculations of angular depen-

$$
\begin{aligned}
& H_{\mathrm{c} 2}(\theta, T) \\
& =-\frac{\hbar c}{2 e} \frac{\left(\alpha_{1}(T) \alpha_{2}(T)-\varepsilon^{2}\right)}{\left.\frac{\hbar_{1}(T)}{m_{2}}\left(\cos ^{2} \theta+\frac{m_{2}}{m_{2}^{2}} \sin ^{2} \theta\right)^{1 / 2}+\frac{\alpha_{2}(T)}{m_{1}}\left(\cos ^{2} \theta+\frac{m_{1}}{m_{1}^{l}} \sin ^{2} \theta\right)^{1 / 2}+\frac{8 \varepsilon \varepsilon_{1}}{\hbar^{2}}\right]} .
\end{aligned}
$$

The above expression is essentially the same as obtained by Gurevich [26] in a similar study making use of Usadel equations. We thus infer that the effective mass replacement introduced above is a reasonable approximation for the moderately anisotropic $\mathrm{MgB}_{2}$, as an expression similar to Eq. (11) was already derived by Gurevich [26].

At small angles $\theta \ll 1$ we obtain the following equation for upper critical field $H_{\mathrm{c} 2}(\theta, T)$

$\frac{H_{\mathrm{c} 2}(\theta, T)}{H_{\mathrm{c} 2}^{\perp}(T)}-1 \simeq \frac{\left(T-T_{\mathrm{c} 2}\right)\left(1-\frac{m_{1}}{2 m_{1}^{c}}\right)+\delta\left(T-T_{\mathrm{c} 1}\right)\left(1-\frac{m_{2}}{2 m_{2}^{c}}\right)}{\left(T-T_{\mathrm{c} 2}\right)+\delta\left(T-T_{\mathrm{c} 1}\right)+8 \varepsilon^{2} \delta \eta T_{\mathrm{c}}} \theta^{2}$,

where we introduced dimensionless parameters as in [13]: $\delta=m_{1} \gamma_{1} / m_{2} \gamma_{2}$ is the parameter which is determined by the ratio of masses in different bands, $\eta=m_{2} \gamma_{2} T_{\mathrm{c}} \varepsilon_{1} / \hbar^{2} \varepsilon$ is the intergradient interaction parameter in energy units. At large tilt angles, i.e., $\cos \theta \gg 1$, upper critical field is given by the following formula:

$$
\begin{aligned}
\frac{H_{\mathrm{c} 2}(\theta, T)}{H_{\mathrm{c} 2}^{\|}(T)}-1 \simeq & -\frac{1}{2}\left[\delta\left(T-T_{\mathrm{c} 1}\right)\left(\frac{m_{2}^{c}}{m_{2}}\right)^{1 / 2}+\left(T-T_{\mathrm{c} 2}\right)\left(\frac{m_{1}^{c}}{m_{1}}\right)^{1 / 2}\right]\left(\frac{\pi}{2}-\theta\right)^{2}\left[\delta\left(T-T_{\mathrm{c} 1}\right)\left(\frac{m_{2}}{m_{2}^{c}}\right)^{1 / 2}+\left(T-T_{\mathrm{c} 2}\right)\left(\frac{m_{1}}{m_{1}^{c}}\right)^{1 / 2}\right. \\
& \left.+8 \varepsilon^{2} \delta \eta T_{\mathrm{c}}+\frac{1}{2}\left[\delta\left(T-T_{\mathrm{c} 1}\right)\left(\frac{m_{2}^{c}}{m_{2}}\right)^{1 / 2}+\left(T-T_{\mathrm{c} 2}\right)\left(\frac{m_{1}^{c}}{m_{1}}\right)^{1 / 2}\right]\left(\frac{\pi}{2}-\theta\right)^{2}\right]^{-1} .
\end{aligned}
$$

dence using quasi-classical Uzadel equations were performed by Golubov and Koshelev [24]. Anisotropy effects in the framework of above presented two-band GL theory [13] was calculated very recently by Udomsamuthirun et al. [17]. However, in this work masses in different bands were taken to be the same and different character of effective masses in different bands was not taken into account. Calculations of the Fermi surface of $\mathrm{MgB}_{2}$ reveals that we have a four-sheet character of the surface [25]. From this point of view, it seems natural to consider two-band GL theory with different anisotropy of masses in different bands. The method presented above for the calculation of the upper critical field $H_{\mathrm{c} 2}$, is the same also in this case. To proceed, one replaces the effective masses $m_{1}$ and $m_{2}$ by an angular dependence

$\frac{1}{m_{i}} \Rightarrow \frac{1}{m_{i}}\left(\cos ^{2} \theta+\frac{m_{i}}{m_{i}^{c}} \sin ^{2} \theta\right)^{1 / 2}$,

in an approximate way, even though the effective masses in an anisotropic superconductor are tensor quantities.

In the vicinity of the critical temperature $T_{\mathrm{c}}$, expression for the upper critical field $H_{\mathrm{c} 2}$ can now be expressed as

\section{Results and discussion}

We now present our numerical results for the angle dependence of $H_{\mathrm{c} 2}(\theta, T)$ calculated for the material parameters of $\mathrm{MgB}_{2}$. We also compare and discuss our results with available experiments. As follows from Eq. (12), if each band has the same mass anisotropy $\left(m_{1} / m_{1}^{c}=\right.$ $\left.m_{2} / m_{2}^{c}\right)$, due to intergradient interaction $H_{\mathrm{c} 2}(\theta, T)$ deviates from elliptic dependence. Expression for the upper critical field $H_{\mathrm{c} 2}(\theta, T)$ shows that the deviation grows with the disparity between $m_{1} / m_{1}^{c}$ and $m_{2} / m_{2}^{c}$. On the other hand, disparity in two-band GL theory depends on the temperature. Thus, it increases away from $T_{\mathrm{c}}$. Examples of angular dependence of upper critical field at low and high temperatures are presented in Fig. 1. In this figure, the solid line corresponds to single-band GL elliptic law and the dotted line corresponds to two-band GL calculations with mass anisotropy. Due to contributions from the $\pi$-band (which corresponds to the band with critical temperature $T_{\mathrm{c} 2}=10 \mathrm{~K}$, see below), one can see significant deviation from single-band anisotropic model at high temperatures. As input parameters we have used the values $T_{\mathrm{c} 1}=20 \mathrm{~K}$, 
$T_{\mathrm{c} 2}=10 \mathrm{~K}$, obtained from various experiments on heat capacity, thermal expansion, and thermal conductivity [27] in $\mathrm{MgB}_{2}$, which yields $\varepsilon^{2} / \gamma_{1} \gamma_{2}=(3 / 8) T_{\mathrm{c}}^{2}$. Note that, based on the experimental results it has been argued in the extensive review by Buzea and Yamashita [28] that $T_{\mathrm{c} 1} / T_{\mathrm{c} 2} \simeq 2$. We have also used $\varepsilon_{1}=0.0976$ which corresponds to $\mathrm{MgB}_{2}$ employed in previous calculations [11-13]. As shown by Mazin et al. [29] the inter-band impurity scattering in $\mathrm{MgB}_{2}$ is small, even in low quality samples, therefore in our calculations the value for $\varepsilon_{1}$ is not sample dependent. According to microscopic calculations the ratio of masses in different bands is $\delta=3$. We also use the following data for mass anisotropies in different bands $\left(m_{1} / m_{1}^{c}=0.03, m_{2} / m_{2}^{c}=1.3\right)$. Similar values were also used by Miranovich et al. [30] in a related calculation. For these parameters and for the intermediate angles $\theta$ we obtain the following approximate expression for $H_{\mathrm{c} 2}(\theta, T)$ :
In Fig. 2 we show the temperature dependence of the anisotropy parameter of upper critical field $\gamma_{\mathrm{c} 2}(T)=$ $H_{\mathrm{c} 2}^{\|} / H_{\mathrm{c} 2}^{\perp}$, which can be calculated as

$$
\gamma_{\mathrm{c} 2}(T)=\sqrt{\frac{m_{2}^{c}}{m_{2}}} \frac{\left(T-T_{\mathrm{c} 2}\right)+\delta\left(T-T_{\mathrm{c} 1}\right)+8 \varepsilon^{2} \delta \eta T_{\mathrm{c}}}{\sqrt{\frac{m_{1} m_{2}^{c}}{m_{2} m_{1}^{c}}}\left(T-T_{\mathrm{c} 2}\right)+\delta\left(T-T_{\mathrm{c} 1}\right)+8 \varepsilon^{2} \delta \eta T_{\mathrm{c}}} .
$$

In this figure, the solid line is the two-band GL calculation result, based on the expression given in Eq. (16). Experimental data for single crystals of $\mathrm{MgB}_{2}$ of Angst et al. [19] are given by the full circles, Shi et al. [21] results by squares, and Lyard et al. [22] results by triangles. It is necessary to note here that even though the experiments have systematic differences amongst themselves, there seems to be a general decreasing trend in $\gamma_{\mathrm{c} 2}$ as $T_{\mathrm{c}}$ is approached. In contrast to the single-band GL theory (see Eqs. (9) and (10)), the two-band GL theory evidently yields the

$$
\begin{aligned}
\frac{H_{\mathrm{c} 2}(\theta, T)}{H_{\mathrm{c} 2}^{\|}(T)}-1 \simeq & {\left[\delta\left(T-T_{\mathrm{c} 1}\right)\left(\left(\frac{m_{2}}{m_{2}^{c}}\right)^{1 / 2}-\frac{1}{2}\left(\frac{m_{2}^{c}}{m_{2}}\right)^{1 / 2}\right)+\left(T-T_{\mathrm{c} 2}\right)\left(\left(\frac{m_{1}}{m_{1}^{c}}\right)^{1 / 2}-\frac{1}{2}\left(\frac{m_{1}^{c}}{m_{1}}\right)^{1 / 2}\right)\right] \cos ^{2} \theta\left[\left(\delta\left(T-T_{\mathrm{c} 1}\right)\left(\frac{m_{2}}{m_{2}^{c}}\right)^{1 / 2}\right.\right.} \\
& \left.\left.+\left(T-T_{\mathrm{c} 2}\right)\left(\frac{m_{1}}{m_{1}^{c}}\right)^{1 / 2}\right)\left(\sin ^{2} \theta+\frac{1}{2} \frac{\delta\left(T-T_{\mathrm{c} 1}\right)\left(\frac{m_{2}^{c}}{m_{2}}\right)^{1 / 2}+\left(T-T_{\mathrm{c} 2}\right)\left(\frac{m_{1}^{c}}{m_{1}}\right)^{1 / 2}}{\delta\left(T-T_{\mathrm{c} 1}\right)\left(\frac{m_{2}}{m_{2}^{c}}\right)^{1 / 2}+\left(T-T_{\mathrm{c} 2}\right)\left(\frac{m_{1}}{m_{1}^{c}}\right)^{1 / 2}} \cos ^{2} \theta\right)+8 \varepsilon^{2} \delta \eta T_{\mathrm{c}}\right]^{-1}
\end{aligned}
$$

As we observe from Fig. 1 and Eq. (15), at high temperatures maximum deviation from the single-band theory is achieved around $\theta \sim 77^{\circ}$. At low temperatures deviation from the single-band effective mass behavior is small (Fig. 1).

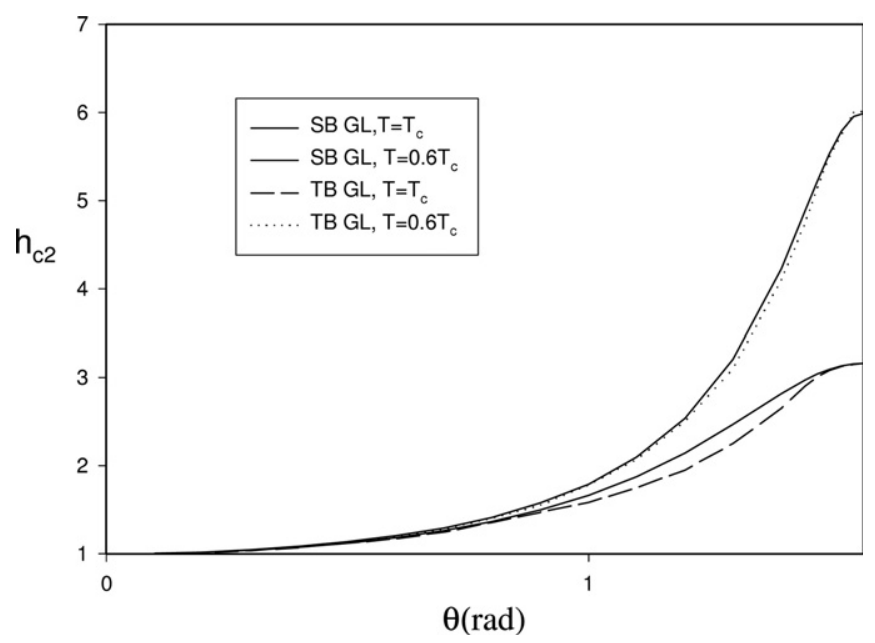

Fig. 1. Angular dependence of the upper critical field $h_{\mathrm{c} 2}=H_{\mathrm{c} 2}(\theta, T) / H_{\mathrm{c} 2}^{\perp}$ at high $\left(T=0.9 T_{\mathrm{c}}\right)$ and low temperatures $\left(T=0.6 T_{\mathrm{c}}\right)$ : full line anisotropic single-band GL theory, dotted line anisotropic two-band GL theory. experimentally observed behavior in $\gamma_{\mathrm{c} 2}$ as a temperature dependent parameter.

The enhanced deviation from single-band GL theory Eq. (9) can be characterized by the parameter $\max _{\theta}(1-A)$, where $A=H_{\mathrm{c} 2}(\theta, T) / H_{\mathrm{c} 2}^{\mathrm{sb}}(\theta, T)$ is the ratio of the upper critical field in the two-band and single-band models. The

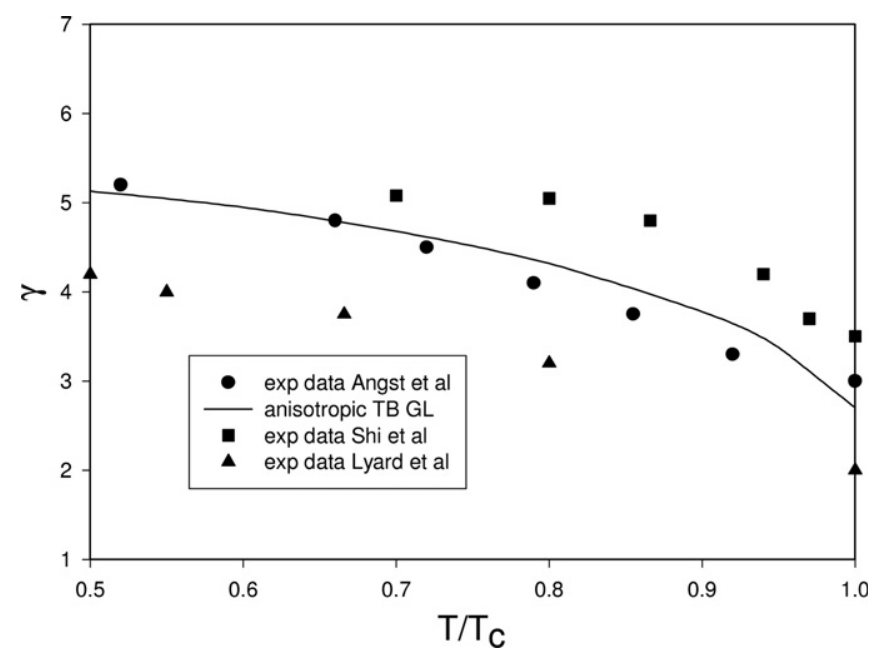

Fig. 2. Temperature dependence of anisotropy parameter $\gamma_{\mathrm{c} 2}$ as a function of temperature. The solid line is anisotropic two-band GL calculations, full circles experimental data of Angst et al. [19], squares experimental data of Shi et al. [21], and triangles experimental data of Lyard et al. [22]. 


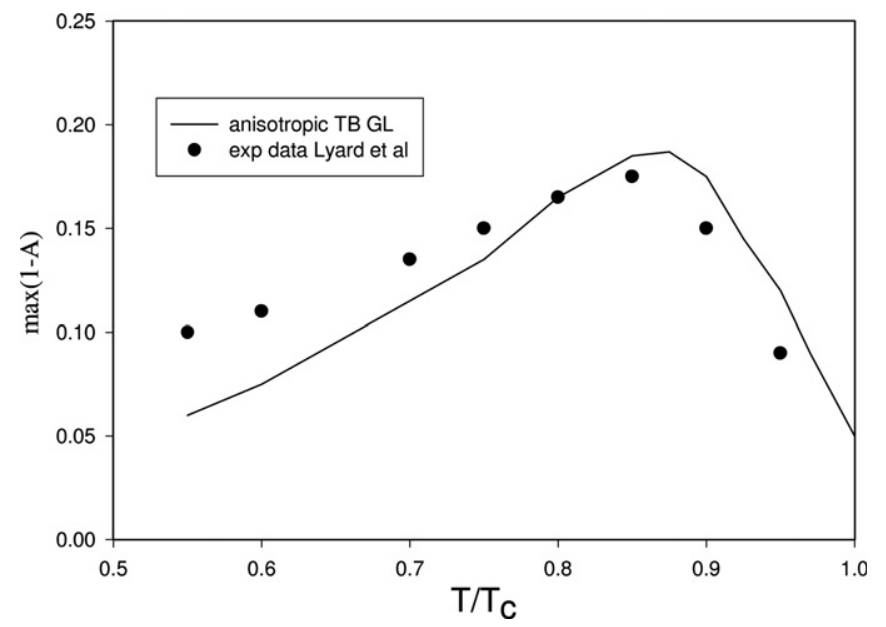

Fig. 3. Temperature dependence of deviation from single-band GL theory, full circles experimental data of Rydh et al. [20].

temperature dependence of this parameter is presented in Fig. 3. The result of the two-band GL calculations is given by the solid line, while experimental data of Rydh et al. [20] are presented by full circles. As seen from Fig. 3 at low temperatures deviation is small and with increasing temperature it first increases and as $T_{\mathrm{c}}$ is approached it starts to decrease. At $T / T_{\mathrm{c}} \sim 0.9$ the deviation parameter reaches maximum $(\sim 0.2)$. As shown by our calculations, the twoband GL yields qualitative and quantitative agreement with experimental data. In particular, in contrast to the results of Dao and Zhitomirsky [32], inclusion of intergradient interaction correctly describe low temperature behavior of deviation parameter.

It is instructive to estimate the region of validity of the GL theory applied to $\mathrm{MgB}_{2}$ within our approach. Due to the large difference in anisotropy, $c$-axis coherence length of second (weak) band $\xi_{2}^{c}=-\frac{\hbar^{2}}{4 m_{2}^{c} \alpha_{2}(T)}$ is much larger than coherence length of first (strong) band $\xi_{1}^{c}=-\frac{\hbar^{2}}{4 m_{1}^{c} \alpha_{1}(T)}$. In the case of common superconductivity, effective coherence length will be $\xi^{c}<\xi_{2}^{c}$ at low temperatures. Applicability of Ginzburg-Landau approach is determined by the condition: $\xi^{c} \gg \xi_{2}^{c}$ which is equivalent to (see Eq. (8))

$$
-\frac{\frac{\hbar^{2}}{4}\left(\frac{\alpha_{1}(T)}{m_{2}}+\frac{\alpha_{2}(T)}{m_{1}}\right)+2 \varepsilon \varepsilon_{1}}{\left(\alpha_{1}(T) \alpha_{2}(T)-\varepsilon^{2}\right)}>\frac{\hbar^{2}}{4 m_{2}^{c} \alpha_{2}(T)} .
$$

Using dimensionless parameters, the above expression can be written as

$$
-\frac{\left(\tau-\frac{T_{\mathrm{c} 2}}{T_{\mathrm{c}}}\right)+\delta\left(\tau-\frac{T_{\mathrm{c} 1}}{T_{\mathrm{c}}}\right)+8 \eta \delta \varepsilon^{* 2}}{\left(\left(\tau-\frac{T_{\mathrm{c} 1}}{T_{\mathrm{c}}}\right)\left(\tau-\frac{T_{\mathrm{c} 2}}{T_{\mathrm{c}}}\right)-\varepsilon^{* 2}\right)}>\frac{\frac{m_{2}}{m_{2}^{c}}}{\left(\tau-\frac{T_{\mathrm{c} 2}}{T_{\mathrm{c}}}\right)},
$$

where $\tau=T / T_{\mathrm{c}}$ and the other parameters have been defined after Eq. (13). Using the numerical values of these parameters, we can show that the violation of the above condition occurs for $T \sim 27 \mathrm{~K}$. This means that, the temperature region of applicability of GL theory is much wider than the narrow region suggested by Golubov and Koshelev [24]. It appears that Golubov and Koshelev [24] approach corre- sponds to an effective single-band GL theory. In their paper, the ratio of order parameters is temperature and field-independent. In our approach, however, the ratio of order parameters is temperature and field-dependent, i.e., $\Psi_{1}(x)=C \Psi_{2}(x)$, where $C=-\frac{\varepsilon-\varepsilon_{1} \delta}{\frac{h^{2}}{4 m_{1}} \delta+\alpha_{1}(T)}$, (see the relevant equations given by Askerzade [13]).

As stated above, all coefficients $\alpha$ and $\beta$ in the GL model are field-independent. Other generalizations of the present model are possible by introducing field-dependent parameters $\alpha$ and $\beta$ [31]. Inclusion of field-dependent coefficients in the framework of two-band GL is a subject for future investigations. Another interesting problem is the study of $H_{\mathrm{cl}}(\theta, T)$ and $\lambda(\theta, T)$ dependence in the framework of anisotropic two-band GL theory.

We conclude that the anisotropic two-band GL theory explains the deviation from elliptic law for the angular dependence of $H_{\mathrm{c} 2}(\theta, T)$. We have used the two-band GL theory with two different mass anisotropy in different bands. The deviation from single-band GL theory is maximum in the vicinity of $T_{\mathrm{c}}$. A compact formula for the $\gamma_{\mathrm{c} 2}(T)=H_{\mathrm{c} 2}^{\|} / H_{\mathrm{c} 2}^{\perp}$ is found using anisotropic two-band model. Temperature dependence of the upper critical field of two-band superconductors $\gamma_{\mathrm{c} 2}(T)$ is in good agreement with experimental data for $\mathrm{MgB}_{2}$.

\section{Acknowledgments}

I.N.A. thanks the Physics Department of Bilkent University for hospitality and TUBITAK for financial support. B.T. acknowledges support from TUBITAK (106T052) and TUBA.

\section{References}

[1] J. Nagamatsu, N. Nakagawa, T. Muranaka, Y. Zenitani, J. Akimitsu, Nature 410 (2001) 63.

[2] J. Kortus, I.I. Mazin, K.D. Belashchenko, V.P. Antropov, L.L. Boyer, Phys. Rev. Lett. 86 (2001) 4656.

[3] A.Y. Liu, I.I. Mazin, J. Kortus, Phys. Rev. Lett. 87 (2001) 87005.

[4] S.L. Budko, G. Lapertot, C. Petrovic, C.E. Cunningham, N. Anderson, P.C. Canfield, Phys. Rev. Lett. 86 (2001) 1877.

[5] T. Yildirim, O. Gülseren, J.W. Lynn, C.M. Brown, T.J. Udovic, Q. Huang, N. Rogado, K.A. Regan, M.A. Hayward, J.S. Slusky, T. He, M.K. Haas, P. Khalifah, K. Inumaru, R.J. Cava, Phys. Rev. Lett. 87 (2001) 037001.

[6] X.K. Chen, M.J. Konstantinovic, J.C. Irwin, D.D. Lawrie, J.P. Franck, Phys. Rev. Lett. 87 (2001) 157002.

[7] F. Giubileo, D. Roditchev, W. Sacks, R. Lamy, D.X. Thanh, J. Klein, S. Miraglia, D. Fruchart, J. Marcus, P. Monod, Phys. Rev. Lett. 87 (2001) 177008.

[8] F. Bouquet, R.A. Fisher, N.E. Phillips, D.G. Hinks, J.D. Jorgensen, Phys. Rev. Lett. 87 (2001) 047001.

[9] S.V. Shulga, S.-L. Drechsler, G. Fuchs, K.-H. Muller, K. Winzer, M. Heinecke, K. Krug, Phys. Rev. Lett. 80 (1998) 1730.

[10] S.V. Shulga, S.-L. Drechsler, H. Echrig, H. Rosner, W. Pickett, 2001, cond-mat/0103154, unpublished.

[11] I.N. Askerzade, A. Gencer, N. Guclu, Supercond. Sci. Technol. 15 (2002) L13.

[12] I.N. Askerzade, A. Gencer, N. Guclu, A. Kiliç, Supercond. Sci. Technol. 15 (2002) L17. 
[13] I.N. Askerzade, Physica C 397 (2003) 99.

[14] M.E. Zhitomirsky, V.H. Dao, Phys. Rev. B 69 (2004) 054508.

[15] H. Doh, M. Sigrist, B.K. Cho, S.-I. Lee, Phys. Rev. Lett. 83 (1999) 5350 .

[16] I. Affleck, M. Frank, M.H. Sharifzadeh Amin, Phys. Rev. B 55 (1997) R704.

[17] P. Udomsamuthirun, A. Changjan, C. Kumvongsa, S. Yoksan, Physica C 434 (2006) 62.

[18] M. Tinkham, Introduction to Superconductivity, McGraw-Hill, New York, 1996, p. 454.

[19] M. Angst, R. Puzniak, A. Wisniewski, J. Jun, S.M. Kazakov, J. Karpinski, J. Roos, H. Keller, Phys. Rev. Lett. 88 (2002) 167004.

[20] A. Rydh, U. Welp, A.E. Koshelev, W.K. Kwok, G.W. Crabtree, R. Brusetti, L. Lyard, T. Klein, C. Marcenat, B. Kang, K.H. Kim, K.H.P. Kim, H.-S. Lee, S.-I. Lee, Phys. Rev. B 70 (2004) 132503.

[21] Z.X. Shi, M. Tokunaga, T. Tamegai, Y. Takano, K. Togano, H. Kito, H. Ihara, Phys. Rev. B 68 (2003) 104513.

[22] L. Lyard, P. Samuely, P. Szabo, T. Klein, C. Marcenat, L. Paulius, K.H.P. Kim, C.U. Jung, H.-S. Lee, B. Kang, S. Choi, S.-I. Lee, J. Marcus, S. Blanchard, A.G.M. Jansen, U. Welp, G. Karapetrov, W.K. Kwok, Phys. Rev. B 66 (2002) 180502(R).
[23] M. Zehetmayer, M. Eisterer, J. Jun, S.M. Kazakov, J. Karpinski, A. Wisniewski, H.W. Weber, Phys. Rev. B 66 (2002) 052505.

[24] A.A. Golubov, A.E. Koshelev, Phys. Rev. B 68 (2003) 104503; A.A. Golubov, A.E. Koshelev, Phys. Rev. Lett. 92 (2004) 107008

[25] H.J. Choi, D. Roundy, H. Sun, M.L. Cohen, S.G. Louie, Phys. Rev. B 66 (2002) 020513(R).

[26] A. Gurevich, Phys. Rev. B 67 (2003) 184515.

[27] N.V. Anshukova et al., Phys. Solid State 45 (2003) 1207; N.V. Anshukova et al., Phys. Solid State 45 (2003) 6; Y. Wang, T. Plackowski, A. Junod, Physica C 355 (2001) 179.

[28] C. Buzea, T. Yamashita, Supercond. Sci. Technol. 14 (2001) R115.

[29] I. Mazin, O.K. Andersen, O. Jepsen, O.V. Dolgov, J. Kortus, A.A. Golubov, A.B. Kuzmenko, D. van der Marel, Phys. Rev. Lett. 89 (2002) 107002.

[30] P. Miranovic, K. Machida, V.G. Kogan, J. Phys. Soc. Jpn. 72 (2003) 221.

[31] M. Eisterer, M. Zehetmayer, H.W. Weber, J. Karpinski, Phys. Rev. B 72 (2005) 134525.

[32] V.H. Dao, M.E. Zhitomirsky, Eur. Phys. J. B44 (2005) 183. 